\title{
Future Mobile Health Systems: Designing Personal Mobile Applications to Assist Self Diagnosis
}

\author{
Dina Ahmad, Andreas Komninos, Lynne Baillie \\ Glasgow Caledonian University \\ 70 Cowcaddens Rd. \\ Glasgow G4 OBA \\ +441413313095 \\ dahmad10@caledonian.ac.uk, [ako2, lba2]@gcal.ac.uk
}

\begin{abstract}
In Europe we live in an increasingly ageing society and solutions that help that ageing society to self care in context are a major goal for the EU and UK governments. Given this climate coupled with the fact that more than $80 \%$ of people aged 35-54 own a mobile phone (National Statistics, 2003) we believe that developing an easy to use application for self diagnosis may be of great use to this age group as they grow older. In our paper we present how we designed one such mobile application, targeted to $40-55$ years olds with declining eyesight. Our system enables users to undertake self diagnosis via their mobile phone. This article reports our findings on the challenges of interface design for this particular age group.
\end{abstract}

\section{Categories and Subject Descriptors}

H.5.2 [User Interfaces]: Prototyping, Screen Design, Usercentred Design

\section{General Terms}

Design, Human Factors

\section{Keywords}

Mobile application design, Mobile healthcare

\section{INTRODUCTION}

Technology is frequently designed to draw people's attention to specific information in an attempt to change what they do or think [1]. In his book, Fogg suggests that persuasive technology can be used to change people's behaviours in a non-commercial domain such as preventative healthcare [2].

Mobile phones are increasingly being used by people of all ages and are becoming an accepted part of people's daily lives. According to the Office for National Statistics, nearly $90 \%$ of people living in the UK between the ages of 15 and 34 owned or use a mobile phone [3]. The ownership percentages of people in the higher age groups are a little lower, about $80 \%$ of people aged 35-54 owned a mobile phone in 2003, compared to $74 \%$ in 2001.
Even though older users are increasingly using mobile phones Kurniawan et al found that older people encounter a range of inhibitors whilst doing so. For example, displays, buttons and characters which are too small and difficult to see, unclear menu's and user manuals [4]. However, Abascal [5] claims that there is no evidence to support the known clichés: "older people reject technology", and says that rejection frequently come from low quality of the interface rather than a lack of acceptance. There are many factors which could affect the use of technology amongst older adults, however some noted by Ogozalek [6] are hearing difficulties and vision related problems. We therefore decided to address the needs of users who may be experiencing a decline in their vision common to old age. One type of decline is Presbyopia (from the Greek word "presbys", meaning "old person" and "opthalmos" meaning "eye"). Presbyopia is a condition whereby the eye exhibits a progressively diminished ability to focus on near objects with age and which affects the majority of people over 40 , requiring them to use "reading glasses".

The project's aim was to investigate how older adults with declining eyesight, aged between 40-55 would respond to a self-diagnosis system on their mobile device, designed using requirements gathered from other adults in the same target group. We used user centered design techniques and involved users in the prototyping, designing and evaluation phases. Our primary objectives were:

- To identify the needs of older adults aged 40-55 with Presbyopia and to involve such adults in the design of the application

- To design a mobile application using published interface design guidelines for the elderly

- To undertake an evaluation of the system with target end users

Secondary to the main objectives mentioned above, we ultimately would like to address the following objectives, through the full implementation and evaluation of a mobile health monitoring system:

- To investigate how older adults aged 40-55 with Presbyopia monitor their health currently

- To identify if older adults are more likely to use their mobile device to monitor their health (provided interface requirements are met) than using traditional methods 


\section{METHODOLOGY}

There were three main phases during the project's time scale requirements gathering, design and development and evaluation. All of these phases are discussed in this section.

\subsection{Requirements Gathering}

We first of all interviewed and ran focus groups with small groups of 4 adults aged 40-55 with Presbyopia. We wanted to investigate what they think about the design of their current mobile phone interface. This is important as Melenhorst [7] and Abascal [5] found that current interfaces have not been designed to satisfy this age group's ability or needs and that this was the main reason for rejection of other features on mobile phones. The result as found by Coates [8]is that older adults limit their interaction with mobile phones to calling or sending text messages. Another important group that we interviewed during this phase was the health professionals involved in diagnosing Presbyopia e.g. Opticians and GPs.

\subsection{Design \& Prototype Development}

The main purpose of the design phase is to produce the application's user interface in line with the user's needs, and to achieve this, paper-prototyping was selected. This technique was chosen to give the users the opportunity to express their needs in an informal way. There are many advantages to using this technique for this project. First, paper prototyping allows early software requirements to be refined as the users will be looking, interacting and modifying with printed representations of the application [9]. Second, paper prototyping was used to test the applications usability; this would ultimately measure the success or failure of the initial design [9]. Third this technique was selected because in the past we [10] have found that this is a useful technique for initiating co-design with users whilst design mobile applications. This has also been reported by other researchers such as Weiss [11]. A small number of people are needed to create a design using paper prototyping [9], thus, for our early stage work, 4 people within our identified target group participated in creating and critiquing the application user interface design in two paper prototyping sessions.

The next stage of the project's life cycle was to produce a functional software prototype of the application. The mobile phone application was implemented using Java for mobile devices (J2ME). The J2ME language was selected as the preferred programming language to develop the application as it allows the prototype to run on multiple devices without requiring re-compilation and thus would allow us to evaluate the software across several platforms.

\subsection{Evaluation}

We used three task scenarios (Task 1, Task 2a, and Task 2b); these task scenarios mapped directly to the main pathways through the application. We decided upon this technique as it had proved to be useful in the past for the evaluation of mobile applications [10][12]. We also used the Think Aloud technique as outlined by Nielsen et al [13]. Finally, we also conducted a structured interview. By combining these three very different techniques we hoped to capture as many HCI issues as possible.

\section{RESULTS}

In this section we highlight the main findings from each phase of our project.

\subsection{Requirements Gathering}

In the focus groups the participants were asked to speak about their current use of their mobile phone. They were particularly asked about their use of any of the applications. It was found that they all had several issues to relate. The main points that were made by several or all of the participants were the following:

- Problems with the use of the calendar on the mobile phone as the numbers were too small

- Difficult to see contacts details on the screen

- Non-use of the internet on the mobile because of the interaction problems such as excessive scrolling.

- Difficult to learn how to undertake certain tasks on the mobile device, e.g. withholding their number.

The discovery of these issues then helped to set the stage for the next part of the session which was the paper prototyping session. In this session, the participants were given paper props (a life-size cardboard cutout of a Nokia N95 phone and appropriately-sized blank paper cards to represent the screen) and were left to design an interface according to the task, without intervention from the designers.

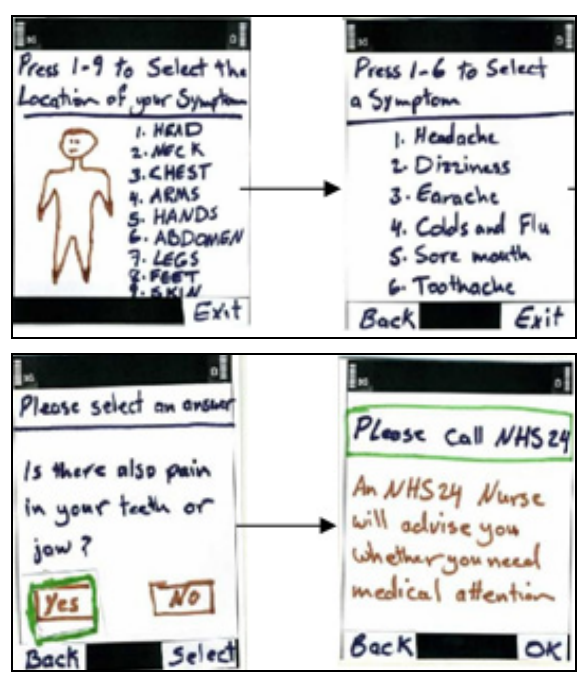

Figure 1: Some samples from paper prototyping design sessions

During the prototyping phase, a common set of design "guidelines" was established, which the participants felt would help them to overcome the difficulties they had found previously when using a mobile phone application. The following interface design guidelines were produced by the participants:

- Use Black text for writing

- Use a White background

- Use the number key pad as shortcuts to navigate

- Use a large, readable font

- Keep the consistency of all pages throughout the application design

- Keep the design as simple to navigate as possible

Hawthorn [14] undertook extensive studies with older people as regards the design of internet sites. His research produced very similar design guidelines to the one we found during our studies e.g. use dark characters on a light background, use a large font etc. Some samples of the outcome of the paper prototyping session can be seen in Figure 1. 


\subsection{Design}

The paper prototype design was then turned into a high fidelity prototype to evaluate the findings found in the design phase and therefore representing the user interface desired by participants in a more practical environment. Figure $2 \mathrm{a}$ shows the first screen of the prototype.

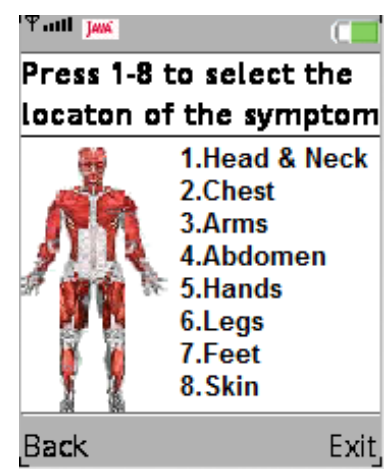

(a)

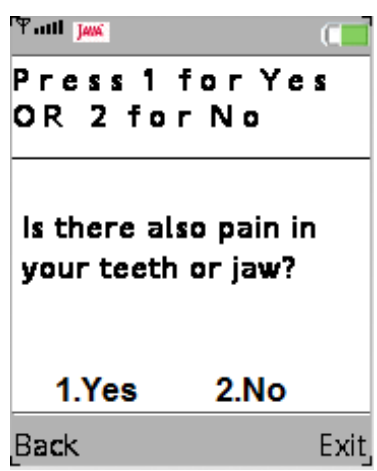

(b)
Figure 2: Functional Prototype Screenshots

The implementation facilitates the user selection by using the phone's Number Key Pad and the phone's Soft Key Pad. The application starts with an image of a human body and a list of numbers from $1-8$, each number corresponds to a human body part. The system then asks the user to press a number to locate the symptom in their body that they are having, this is the first point of interaction between the user interacts and the system. The System then continues in asking the users to select a function using shortcuts via the number key pad. As previously mentioned in 3.1, the participants were keen to design a system that was simple and easy to interact with. This simplistic type of design can also be seen in the selection for "Yes" or "No" in Figure $2 b$.

In addition, the colours adopted for this prototype i.e. predominantly black and white were selected because of feedback from users and other research studies (e.g. [14]).

The system allows the user to go back to the previous screen by pressing the Back button on the phone's left soft key pad as well as allowing the user to quit the application at any time by using the Exit button on the right key on the same key pad.

The first iterations of the prototype development were carried out using a Nokia N95 mobile phone as the target platform (see Appendix G). The N95 was ideal for this project as it has a relatively large screen display therefore the application was deployed on the same device.

We consulted with NHS24 Scotland, who operate a selfdiagnosis website (www.nhs24.com) and asked for permission to use their content and decision-making algorithms for the application. These were eagerly provided along with further insights to particular points of interest (such as that decision tree outcomes should err on the side of caution, prompting users to consult the NHS in most cases).

\subsection{Evaluation}

The evaluation of the interface design was carried out in three stages: Firstly, the design was validated by an independent HCI expert with the same age characteristics as the target group, who was asked to think-aloud while performing three tasks. This uncovered some minor issues such as the need of a bolder selection frame around the Yes/No options. In response, we changed the design by implementing key shortcuts consistent with the main menu. Secondly, a usability questionnaire was issued to eleven non-expert users within the target group age frame. Of these users, $91 \%$ agreed or strongly agreed that the text was easy to read and $71 \%$ were happy with the combination of black text over a white background. With regard to preference of using numeric keypad shortcuts instead of the "joystick" for making choices, $81 \%$ responded they preferred the shortcuts. All participants either agreed or strongly agreed that the application's screens were easy to navigate through and all participants strongly agreed that the application was intuitive and easy to learn. Despite these extremely positive findings, $63 \%$ of users indicated intent to use the application to diagnose a symptom ("agree") and 36\% were unsure.

The next stage of the evaluation involved a four-week long trial period, during which participants were given the application and were asked to keep diary logs of how often it was used during the period. It is important to stress here that this was a preliminary, limited scope study, aimed to uncover issues and prepare the ground for a longer and more thorough field trial. We recruited five volunteers from the eleven previous participants that were willing to undertake the study and provided them with the application on a SonyEricsson K610i and a set of diary log sheets. The participants were interviewed prior to the trial period to get an indication of their health monitoring habits. It should be clarified at this stage that we use the term "monitoring" to describe the process of seeking healthrelated information that is personally relevant to the user, from a variety of sources (doctors, pharmacists, internet, friends etc.). Two participants indicated that they visited their doctor regularly (once a month and once fortnightly), while three indicated that they did not monitor their health at all. After the trial period was finished, we collected the diary logs and analysed these to observe the frequency of use of the application.

The results we obtained showed an increase of instances where health was monitored when using the application, compared to previous habits, for all but one user (P1). During postexperiment interviews that person indicated that:

"I have a heart condition and I have to visit the doctor twice a month to check my health therefore I already monitor my health regularly and did not find the need to diagnose any symptoms".

The monthly number of times that each participant monitored their health before and after the application use

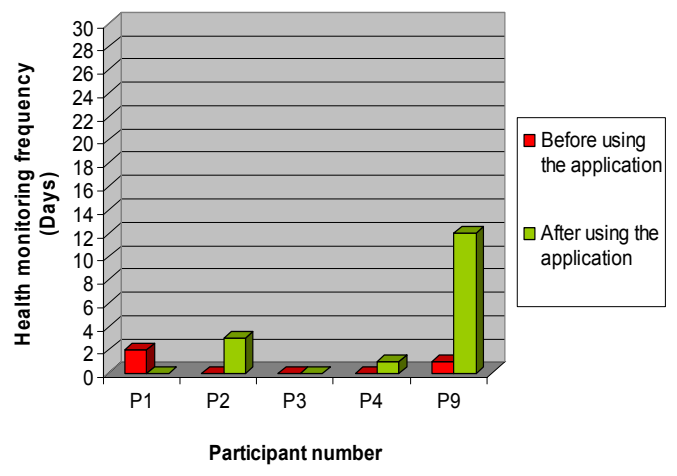

Figure 3: Trial period results summary 
Other users (P2, P4) showed a modest increase, but the results also show that P9 has used the application a lot more than the other users. When asked on the reasons behind the extensive use of the application compared to other users, the participant said:

"In the first week of the trial I was curious to discover the application so I used the application 4 times that week"

The results from P9 were very interesting and lead us to the conclusion that an unforeseen advantage of making the application available to the general population might be that they could expand on their knowledge on general health issues that they may come across in the future. In addition this knowledge could perhaps be useful if a user can diagnose a symptom for family member or a friend.

\section{DISCUSSION AND FUTURE WORK}

Our usage trial of the application design was relatively limited in its extent and the results we found after the trial cannot be generalized across a wider population. Our work is still in progress, however we Naturally, we would like to continue further testing of the interface guidelines by making the application available to a significantly larger subject group. However, the results of the post-design questionnaire survey were very encouraging, indicating that our user-derived design guidelines can help towards the generation of usable interfaces to support the provision of health information to ageing users suffering from presbyopia. A slight concern arose from the percentages of uncertainty regarding the application usage intent. This could have been expected, given the general reservation against mobile technology amongst the target population group. However, our field trials indicated that people who would have otherwise not monitored their health, found the application easy to use and repeatedly used it to find out more facts about their health. We are also hoping to carry out further tests to judge the efficacy of our design guidelines against the current NHS24 website system as viewed on a mobile device.

In our current implementation, we examined the acceptability of a user interface and interaction style as designed through involvement of participants from our target group. We have not, at this stage, evaluated the interaction style and interface against alternative designs, possibly derived from requirements gathering using different age groups. This would be a useful comparison in the future, with the scope to establish universal design guidelines for the development of healthcare-related applications on mobile devices.

In terms of development, our Self-Diagnosis system can be carried further in many ways: it can be first further developed to enable the application to connect to the internet and receive regular updates directly from the NHS 24 website, allowing the NHS to issue general advisories or update decision algorithms. In addition the software can be advanced further to include specific diagnosis to separate body parts or for specific conditions, i.e. a Heart-Disease Self Diagnosis System. In this manner, more precise and detailed algorithms can be used, perhaps coupled with a known user profile or patient record to allow for better accuracy in estimations. Lastly, we would like to test the efficacy of the system with other user groups that require specific needs such as the third age $(60+)$, or people at risk that come from a younger generation.

\section{REFERENCES}

[1] Toscos, T., Faber, A., An, S., Ghandi, M., Chick Clicque: Persuasive Technology to Motivate Teenage Girls to Exercise, in Extended Abstracts of ACM SIGCHI Conference on Human Factors in Computing (CHI'06) 1873-1878, 2006

[2] Fogg, B.J. 2003, Persuasive Technology: Using Computers to Change what we Think and Do, Morgan Kaufmann Publishers, 2003

[3] UK Office of National Statistics: Adult Mobile Phone Ownership or Use: by age, 2001 and 2003: Social Trends 34. Available online at http://www.statistics.gov.uk

[4] Kurniawan, S., Zaphiris, P., Research-derived web design guidelines for older people, $7^{\text {th }}$ ACM SIGACCESS Conference on Computers \& Accessibility, Baltimore, USA, 2005

[5] Abascal, J., Civit, A., Universal Access to Mobile Telephony as a Way to Enhance the Autonomy of Elderly People, Proceedings of the 2001 EC/NSF workshop on Universal Accessibility for Ubiquitous Computing: Providing for the Elderly, Alcacer do Sal, Portugal, 2001

[6] Ogozalek, V.Z., A Comparison of the Use of Text and Multimedia Interfaces to Provide Information for the Elderly, ACM SIGCHI Conference on Human Factors in Computing (CHI'94), Boston, USA, p. 65-71, 1994

[7] Melenhorst, A.S., Rogers, W.A., Caylor, C., The Use Of Communication Technologies By Older Adults: Exploring The Benefits From The User's Perspective, Proceedings of the $45^{\text {th }}$ Annual Meeting of the Human Factors and Ergonomics Society, Minneapolis, USA, 2001

[8] Coates, H. Mobile Phone Users: A Small-Scale Observational Study, 2001. Available online at http://www.aber.ac.uk/media/Students/hec9901.html

[9] Snyder, C. Paper prototyping: The fast and easy way to design and refine user interfaces. Morgan Kaufmann Publishers, London, 2003.

[10] Baillie, L. \& Schatz, R. Exploring Multimodality in the Laboratory and the Field. Seventh ACM International Conference on Multimodal Interfaces, Trento, Italy, 2005

[11] Weiss, S., Handheld Usability, John Wiley \& Sons Publishers, New York, 2002

[12] Lai, J. Facilitating mobile communication with multimodal access to email messages on a cell phone. ACM SIGCHI Conference on Human Factors in Computing Systems, (CHI'04), Vienna, Austria, 2004

[13] Nielsen, J., Mack, R., (eds), Usability Inspection Methods, John Wiley \& Sons Publishers, New York, 1994

[14] Hawthorn, D., Possible implications of aging for interface designers, Interacting with Computers, vol. 12, no. 5, pp. 507-528, 2000 